\title{
On spherically symmetric cosmology in Riemann-Cartan spacetime with spin-density
}

\author{
Larry L Smalley† and Jean P Krisch $\ddagger$ \\ $\dagger$ Department of Physics, University of Alabama in Huntsville, Huntsville, AL 35899, USA \\ †Department of Physics, University of Michigan, Ann Arbor, MI 48109, USA
}

Received 13 July 1993 , in final form 2 July 1994

\begin{abstract}
We develop the average effective energy-momentum tensor for a spherically symmetric cosmology with randomly oriented spin using the improved energy-momentum tensor in the framework of the self-consistent Einstein-Cartan theory with spin-density.

PACS numbers: $9530 \mathrm{~S}, 9880 \mathrm{C}, 4775,0340 \mathrm{G}, 0450$
\end{abstract}

It has been known for nearly two decades in the investigations of Einstein-Cartan (EC) theories that the spin-density of matter heavily influences [1] and could dominate the behaviour of spin aligned fluids for extremely large matter density which may occur at either early or later stages of the universe [2-4]. It was then shown by Hehl, von der Heyde and Kerlick [5] that the general conclusions reached above for aligned spins would also hold for randomly oriented spins which are locally isotropic, i.e. spherically symmetric, Except for Kopczyński [2], however, the spins in these theories were generally associated with the quantum mechanical spin of particles. We feel, however, that it is not necessary to identify the spin-density of a fluid with the spin of an elementary particle. Our approach involving an improved energy-momentum tensor for spinning fluids [6] is similar to the concept of Israel [7], Bailey and Israel [8], Bailey [9] or Kopczyński [5,10], where the cosmological fluid particles, which may be galaxies or clusters of galaxies, have intrinsic but classical spin. Although one could investigate within the context of general relativity (GR) $[11,12]$ the effect of spin on important cosmological issues such as inflation in the early universe [13], the natural arena should be within an EC theory in Riemann-Cartan (RC) spacetime. In fact, recent investigations by Martin et al [14] have indicated that consistency relations $[15,16]$ restrict most interesting spinning fluid configurations in GR even though their work seems to require further analysis. We will find from this work that the lack of spin-squared terms, which do not occur in the GR calculation, limits the range of examples, but we will leave the proof to a future work. A fortiori, in the extensive review of the EC theory, Hehl et al [17] have shown that the field equations and conservation laws for the metric and the angular momentum arise naturally from variational considerations within the EC theory. In RC spaces a fundamental field quantity is the torsion, which is identified with the antisymmetric part of the RC connection $S_{i j}{ }^{k}=\Gamma_{[i j]}{ }^{k}$. The relationship between torsion and physical spin has been clarified and made more preicse [18]. We now know that the spin is directly identified with a natual object in the geometry, namely the trace-free torsion, $\widehat{S}_{i j}^{k}=S_{i j}{ }^{k}+\delta_{[i}^{k} \zeta_{j]}$, where the torsion vector $\zeta_{i}=\frac{2}{3} S_{i x}{ }^{x}$. Within the context of a Lagrangianbased self-consistent EC theory [6], the torsion vector enters the theory depending on the 
form of the fluid constraint. For the case of particle number conservation in the fluid frame, the constraint is given by ${ }^{*} \nabla_{i}\left(\rho u^{i}\right)$ where the 'star' derivative ${ }^{*} \nabla_{i} \equiv \nabla_{i}+2 S_{i x}{ }^{x}$. The torsion field equation then shows that the torsion vector vanishes. For the discussion of stars, gas clouds and galaxies, this type of constraint is probably sufficient; however, for cosmological models, this may not be the case. If the constraint is given by $\nabla_{i}\left(\rho u^{t}\right)$ (which is the direct minimal coupling generalization of the fluid constraint to RC [19]), then the torsion vector remains active; however, its interpretation in the theory is somewhat incomplete, since the torsion field equation can only resolve it in terms of the Lagrange multiplier for the fiuid constraint [18] (as will be seen later on) without a complete solution (model) of the field equations. One interpretation obtained by applying Gauss' law for a region between two spacelike hypersurfaces enclosing all matter leads to mass 'creation' (or annihilation), which may have cosmological significance [6]. This interpretation nevertheless does seem controversial. Since this work involves cosmological questions, we necessarily have used the constraint which keeps the torsion vector active.

Our investigations seem to indicate that the effects of spin-density in cosmological problems such as inflation, final collapse, or even collapsing objects, should be investigated in an RC spacetime [20]. Our averaging treatment of the proper torsion represents an older variation of the microscopic viewpoint of torsion [21]. In fact, using the improved energymomentum tensor with spin-density, Gasperini [22] has demonstrated spin-dominated inflation in the EC theory for a spinning fluid with randomly oriented spin. This result seems most reasonable in comparison with similar conclusions obtained from an earlier ad hoc description of spinning fluids [5] obtained by just adding to the energy-momentum tensor a spin-density contribution based upon a generalization of the special relativistic treatment of Halbwachs [23]. We point out that the improved energy-momentum tensor for a spinning fluid within an EC theory is also a generalization of Halbwachs' classical treatment of spin to RC spacetime, but here we depart from others through the use of a self-consistent, Lagrangian formulation which also treats the thermodynamic properties of the fluid [19]. Thus with these comments in mind, the purpose of this paper is to discuss the changes that occur in the 'average' contribution to the energy-momentum tensor for randomly oriented spins when the self-consistent description of a spinning fluid is used. An expression for the average energy-momentum tensor is then obtained.

The results of the self-consistent EC description of a spinning fluid are the field equations $[6,18,] \dagger$

$$
\begin{aligned}
& G^{(i j)}(\Gamma)-2{ }^{*} T_{k}^{k(i j)}=\kappa T^{i j} \\
& \widehat{S}_{\ell m}{ }^{k}=\frac{1}{2} \kappa \rho S_{\varepsilon m} u^{k} \\
& \zeta_{\ell}=-\kappa \rho \lambda_{2} u_{\ell}
\end{aligned}
$$

where $G^{(i j)}(\Gamma)$ are the symmetric components of the Einstein tensor in RC space;time with torsion $S_{i j}{ }^{k}=\Gamma_{[i j]}{ }^{k}$, where the modified torsion is $T_{i j}{ }^{k} \equiv \widehat{S}_{i j}^{k}-2 \zeta_{[i} \delta_{j]}^{k}, s_{i j}$ is the spin/particle, $\rho$ is the mass density, $\kappa=8 \pi G$, and $\lambda_{2}$ is the Lagrange multiplier for the fiuid constraint. The improved energy-momentum tensor is

$$
T^{i j}=T_{\mathrm{F}}^{i j}+T_{\mathrm{S}}^{i j}
$$

$\dagger$ Our basic notation follows that of [25], except that we use latin letters for spacetime indices. 
where

$$
\begin{aligned}
& T_{\mathrm{F}}^{i j}=[\rho(1+\epsilon)] u^{i} u^{j}+p g^{i j} \\
& T_{\mathrm{S}}^{i j}=2 \rho u^{(i} s^{j) k} \dot{u}_{k}+\stackrel{*}{\nabla}_{\ell}\left[\rho u^{(i} s^{j) \ell}\right]-\rho \omega_{k}{ }^{(i} s^{j) k} .
\end{aligned}
$$

The LHS of equation (1) can be written as the Riemannian Einstein tensor plus torsionsquared correction terms defining the 'spin' correction tensor $-\tau^{i j}$ such that $[5,25]$

$\left.\kappa \tau^{i j}=-4 T^{i k}{ }_{[\ell} T^{j \ell}{ }_{k l}-2 T^{i k \ell} T_{k \ell}^{j}+T^{k \ell i} T_{k \ell}{ }^{j}+\frac{1}{2} g^{i j}\left[4 T_{k}{ }_{[m} T_{p m}^{k m}\right]+T^{k p m} T_{k p m}\right]$.

We then combine equation (7) with (4) to obtain the effective Riemannian energymomentum tensor

$$
T_{\mathrm{R}}^{i j}=T^{i j}+\tau^{i j}
$$

and then find its average for a randomly oriented, spherically symmetric spinning fluid.

The average of the effective energy-momentum tensor is a two-step process. Since a general spherically symmetric tensor takes the form $a u^{i} u^{j}+b g^{i j}$, we can rewrite it in this form for convenience. The average is then easily found. We note that the fluid part is already in this form. The spin parts are then

$$
T_{\mathrm{S}}^{i j}=-\frac{1}{3}\left[4 \Omega_{k \ell} S^{k \ell}+4 \kappa S^{2}-\omega_{k \ell} S^{k \ell}\right] u^{i} u^{j}-\frac{1}{3}\left[\Omega_{k \ell} S^{k \ell}+\kappa S^{2}-\omega_{k \ell} S^{k \ell}\right] g^{i j}
$$

and

$$
\kappa \tau^{i j}=\left[\frac{5}{6} \kappa^{2} S^{2}-6 \zeta^{2}\right] u^{i} u^{j}+\left[\frac{1}{12} \kappa^{2} S^{2}-3 \zeta^{2}\right] g^{i j}
$$

where the spin-density tensor $S^{i j}=\rho s^{i j}$, and $S_{i j} S^{i j}=2 S_{k} S^{k} \equiv 2 S^{2}$ where $S_{k}$ is the spin vector, $\zeta^{k} \zeta_{k}=\zeta^{2}$, and $\Omega_{k \ell}$ is the vorticity tensor in GR [26]†. In taking the average of a spherically symmetric, isotropic system of randomly oriented spins, then for the spin average itself $\left\langle S^{i j}\right\rangle=0$, but for the spin-squared terms $\left\langle S_{i j} S^{i j}\right\rangle \neq 0$. First we note that if we ignore the vorticity, angular velocity and torsion vector terms, we obtain the old EC results for the average of the effective energy-momentum tensor $[5,27] \neq$

$$
\left\langle T_{\mathrm{R}}^{i j}\right\rangle_{\mathrm{NI}}=\left[\rho(1+\epsilon)+p-\frac{1}{2} \kappa S^{2}\right] u^{i} u^{j}+\left[p-\frac{1}{4} \kappa S^{2}\right] g^{i j}
$$

where the subscript NI refers to the results obtained from the non-improved effective energymomentum tensor. We have also set $\left\langle S^{2}\right\rangle=S^{2}$ for convenience.

Another way of looking at the results given by equation (11) is to assume that on average there is no correlation between the randomly oriented spins and the GR vorticity of the congruences associated with 4-velocity, i.e. we have $\left\langle\Omega_{i j} S^{i j}\right\rangle=0$. At first one might

$\uparrow$ See, for example, [26].

$\$$ These are the results for the spin-squared terms obtained by [27], but in order to obtain these results they defined the torsion $S_{i j}{ }^{k}=2 \Gamma_{i i j]^{k}}$, used the ad hoc classical Weyssenhoff relation between torsion and spin $S_{i j}{ }^{k}=S_{i j} u^{k}$, and used $S_{i j} S^{i j}=2 S^{2}$. On the other hand, [5] also used the classical Weyssenhoff relation, but defined the torsion as in this paper, and defined $2 S_{i j} S^{i j}=S^{2}$. Thus in reality, these results are very different from one another because of conflicting definitions. On the surface, the compensating factors of 2 in [27] gives the same look-alike results as the exact calculation of the EC limit found in this paper, but we can not rectify an additional factor of 2 in [5]. 
want to extend this conclusion to the $\omega_{i j} S^{i j}$ terms, but this would be incorrect, since we can write

$$
\omega_{i j} S^{i j}=\omega_{i j}^{(\mathrm{GR})} S^{i j}-\kappa S^{2}
$$

where we have separated a $\mathrm{RC}$ term into a general relativistic term plus torsion related terms. In the form of equation (12) we easily note that the average is non-zero, because of the presence of the spin-squared term. We further argue that the average of the general relativistic term vanishes (similarly to the vorticity-spin term), since it can be written in terms of products of the uncorrelated tetrads (or, equivalently, metric terms) without any torsion terms present. Thus

$$
\left\langle\omega_{i j} S^{i j}\right\rangle=-\kappa S^{2}
$$

Combining this with equations (9) and (10), we find in the self-consistent EC theory the average of the effective energy-momentum tensor

$$
\left\langle\kappa T_{\mathrm{R}}^{i j}\right\rangle_{\mathrm{I}}=\left[\kappa \rho\left(1+\epsilon+\frac{p}{\rho}\right)-\frac{5}{6} \kappa^{2} S^{2}-6 \zeta^{2}\right] u^{i} u^{j}+\left[\kappa p-\frac{7}{12} \kappa^{2} S^{2}-3 \zeta^{2}\right] g^{i j}
$$

where the subscript I refers to the improved results. Note this does not reduce to the old EC results in equation (11), because of the contribution of the angular velocity term in the improved energy-momentum tensor given by equation (6). The effect of the negative contribution of the spin-density to the energy-density is about twice as large as the old EC results. However, because of the torsion vector field equation (3), the torsion vector contributes positive terms to the energy-momentum density. This is not entirely conclusive, since one needs to introduce source fields (e.g. a vector field Lagrangian sensitive to the torsion vector) and then include the contribution of those fields in the energy-momentum tensor as well. The Lagrange multiplier can be removed from equation (3) by solving the constraint field equation from the variation of the Lagrangian [6]. This gives an equation of motion for $\lambda_{2}$

$$
\kappa \rho \dot{\lambda}_{2}=\kappa\left[\rho\left(1+\epsilon+\frac{p}{\rho}\right)+T_{\mathrm{S}}\right]+3\left(\kappa \rho \lambda_{2}\right)^{2}
$$

where $T_{\mathrm{S}}$ is the spin energy density. Equation (15) for $\lambda_{2}$ can be solved within the context of some cosmological model. For the example of constant density $\rho$ and uniform total energy density, the contribution of the torsion vector terms to the average energy-momentum equation (14) is a monotonically increasing function of proper time. Although an unlikely model, it does serve to show that the torsion vector terms and the spin terms act oppositely to one another.

Thus, although we agree with the general conclusion of Gasperini concerning spindominated inflation [22], his result is based upon equation (11), which neglects the angular velocity contributions which would give a much stronger spin effect, as is seen in the average for the self-consistent EC theory given in equation (14).

In conclusion, equation (14) describes the average effective energy-momentum tensor due to the effects of torsion in an RC spacetime. The averaging process could be thought of as the transition from the microscopic to the macroscopic description of a spin field.

In a different approach to cosmological models, Smalley has shown how one can obtain a spacetime-dependent cosmological 'function' in a Weyl spacetime [28]. It has been 
known for some time that in some affine geometries the torsion and Weyl vectors may be proportional [29]. One way to see this is to note that the fluid constraint in an affine geometry will contain an independent connection that can have both torsion and nonmetricity, the trace of the non-metricity being the Weyl vector. The field equations for the torsion vector and the Weyl vector then give the proportionality. The similarities of the results of spacetime geometries with torsion vector or Weyl vector have been pointed out earlier [30]. The linking of the torsion vector to a cosmological function would be interesting. However, the relationship to this work is only suggestive, since one first needs a Lagrangian theory with all the appropriate elements, including source fields as well. We leave this for future work.

Finally, we mention that other approaches to the torsion vector have not been included in this work, such as the possible relationship of torsion and electromagnetism by Hammond [31] or that of Smalley and Krisch [32].

\section{References}

[1] Hehl F W and von der Heyde P 1973 Ann. Inst. H Poincaré A 19179

[2] Kopczyński W 1972 Phys. Lett. 39A 219; 1973 Phys. Lett. 43A 63

[3] Trautman A 1973 Nat. Phys. Sci. 2427

[4] Stewart J and Hájicek P 1973 Nat. Phys. Sci. 24496

[5] Hehl F W, von der Heyde P and Kerlick G D 1974 Phys. Rev. D 101066

Hehl F W 1974 Gen. Rel. Grav. 5491

[6] Ray J R and Smalley L L 1982 Phys. Rev. Lett. 49 1059; 1983 Phys. Rev. Lett. 50 626E; Phys. Rev. D 27 1383

[7] Israel W 1973 Nuovo Cimento 7860

[8] Bailey I and Israel W 1975 Commun. Math. Phys. 4265

[9] Bailey I 1979 Ann. Phys., NY 11976

[10] Kopczyński W 1986 Phys. Rev. D 34352

[11] Ray J R and Smalley L L 1982 Phys. Rev. D 262619

[12] Ray J R, Smalley L L and Krisch J P 1987 Phys. Rev, D 353261

[13] Bedran M L and Vasconcellos-Vaidya 1984 Nuovo Cimento 4173

[14] Martin M A P, Vasconcellos-Vaidya E P and Som M M 1991 Class. Quantum Grav. 82225

[15] Amorim R 1984 Phys. Lett. 104A 259

[16] Ray J R and Smalley L L 1986 Phys. Rev. D 343268

[17] Hehl F W, von der Heyde P, Kerlick G D and Nester J M 1976 Rev. Mod. Phys, 48393

[18] Smalley L L and Ray J R 1986 Gen. Rel. Grav. 18549

[19] Ray J R 1972 J. Math. Phys. 131451

[20] Arkuszewski W, Kopczyński $W$ and Ponomariev V N 1974 Ann. Inst. H Poincaré 21 89; 1975 Commun. Math. Phys. 45183

[21] Hehl F W, McCrea J D, Mielke E W and Ne'eman Y 1989 Found. Phys. 191075

[22] Gasperini M 1986 Phys. Rev. Lett. 562873

[23] Halbwachs F 1960 Théorie relativiste des fuides à spin (Paris: Gauthier-Villars)

[24] Hehle F W 1974 Gen. Rel. Grav. 5491

[25] Schouten J A 1954 Ricci Calculus (Berlin: Springer)

[26] Ehlers J and Kundt W 1962 Gravitation: An Introduction to Current Research ed L Witten (New York: Wiley) pp 49-101

[27] Arkuszewski W, Kopczyński W and Ponomariev V N 1974 Ann. Inst. H Poincaré 2189

[28] Smalley L L 1993 Class. Quantum Grav. 101179

[29] Hehi F W, Lord E A and Smalley L L 1978 Gen. Rel. Grav. 9691

[30] Smalley L L 1986 Phys. Rev. D 333590

[31] Hammond R T 1988 Gen. Rel. Grav. 20813

[32] Smalley L L and Krisch J P 1991 Class. Quantum Grav. 8 1889; 1991 J. Math. Phys. 33 1073; 1992 Int. J. Theor. Phys. 311253 